\title{
Interactions and Reactions of Transition Metal Clusters with the Interior of Single-Walled Carbon Nanotubes Imaged at the Atomic Scale
}

Thilo Zoberbier, ${ }^{\dagger}$ Thomas W. Chamberlain, ${ }^{\ddagger}$ Johannes Biskupek, ${ }^{\dagger}$ Navaratnarajah Kuganathan, ${ }^{\ddagger}$

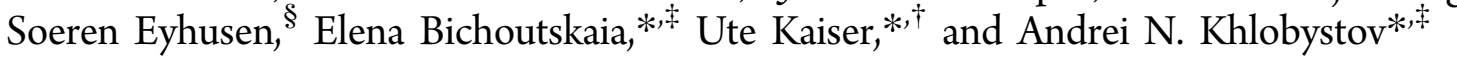

${ }^{\dagger}$ Group of Electron Microscopy of Materials Science, Central Facility for Electron Microscopy, Ulm University, Albert Einstein Allee 11, Ulm 89081, Germany

${ }^{\ddagger}$ School of Chemistry, University of Nottingham, University Park, Nottingham NG7 2RD, United Kingdom

${ }^{\S}$ Carl Zeiss NTS GmbH, Carl-Zeiss-Strasse 56, 73447 Oberkochen, Germany

Supporting Information

ABSTRACT: Clusters of transition metals, W, Re, and Os, upon encapsulation within a single-walled carbon nanotube (SWNT) exhibit marked differences in their affinity and reactivity with the SWNT, as revealed by low-voltage aberration-corrected high-resolution transmission electron microscopy (AC-HRTEM). Activated by an $80 \mathrm{keV}$ electron beam, W reacts only weakly with the SWNT, Re creates localized defects on the sidewall, and Os reacts readily causing extensive defect formation and constriction of the SWNT sidewall followed by total rupture of the tubular structure. AC-HRTEM imaging at the atomic level of structural transformations caused by

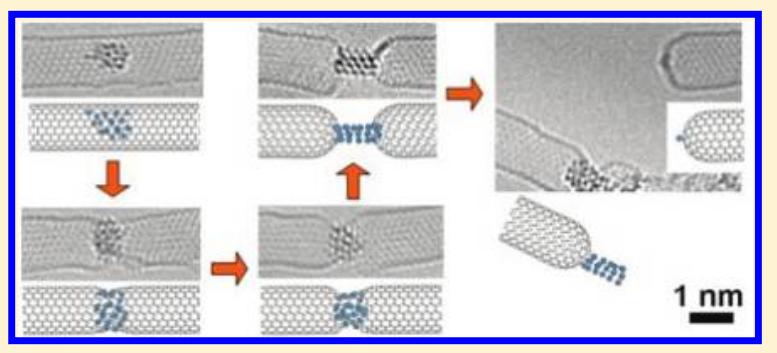
metal-carbon bonding of $\pi$ - and $\sigma$-character demonstrates what a crucial role these types of bonds have in governing the interactions between the transition metal clusters and the SWNT. The observed order of reactivity $\mathrm{W}<\mathrm{Re}<\mathrm{Os}$ is independent of the metal cluster size, shape, or orientation, and is related to the metal to nanotube bonding energy and the amount of electronic density transferred between metal and SWNT, both of which increase along the triad W, Re, Os, as predicted by firstprinciples density functional theory calculations. By selecting the appropriate energy of the electron beam, the metal-nanotube interactions can be controlled (activated or precluded). At an electron energy as low as $20 \mathrm{keV}$, no visible transformations in the nanotube in the vicinity of Os-clusters are observed.

\section{INTRODUCTION}

Transition metals ( $d$-elements) form the largest block of the Periodic Table and offer the widest variety of magnetic, optical, catalytic, and other functional properties. The rich chemistry of transition metals when combined with the mechanical, electric, thermal, and chemical properties of carbon nanostructures, such as single-walled carbon nanotubes (SWNTs), may lead to the generation of new families of functional materials which harness the synergy of the resultant metal-nanocarbon interactions. Recent investigations of metal-SWNT heterostructures have opened new highly promising avenues for applications in catalysis, ${ }^{1}$ hydrogen storage, ${ }^{2}$ and electronic devices. ${ }^{3}$ Therefore, the quest for complete understanding of the nature of bonding between carbon nanotubes and transition metals is becoming increasingly important as illustrated by a recent flurry of theoretical studies on interactions between transition metals and SWNTs. ${ }^{4}$ However, experimental measurements are significantly impeded because of the typical polydispersity of nanotubes (i.e., SWNTs of different lengths, diameters and helicities are present in the same sample), the lack of their intrinsic solubility, and by ubiquitous impurities in SWNT samples (e.g., amorphous carbon, graphitic particles, residual metal catalyst). While conventional spectroscopic methods that integrate over larger volumes (e.g., XPS, Raman, etc.) can be applied for characterizing the bulk physicochemical properties, high-resolution transmission electron microscopy (HRTEM) is now rapidly becoming an excellent local-probe tool for studying chemical reactions in nanotubes by imaging transformations in direct space and real time down to the single-atom level. ${ }^{5}$

Being mechanically, thermally, and chemically very stable, carbon nanotubes primarily act as passive containers for molecules and atoms in previous investigations. Our current study, however, demonstrates that the inner surface of carbon nanotubes can be engaged in interactions and reactions with small clusters of transition metals. We show that the interactions and types of bonding between the SWNT and the metal are determined by the chemical properties of the respective $d$-elements. Subtle differences in the chemical reactivity of the transition metals can have a drastic impact on the resultant transformations of the SWNTs. Our

Received: September 24, 2011

Published: January 17, 2012 
observations are correlated with first-principles density functional theory calculations, which give a basic framework for predicting and designing metal-catalyzed chemical reactions of carbon nanotubes.

\section{RESULTS AND DISCUSSION}

A carbon nanotube has two qualitatively different surfaces: the exterior surface and the interior surface. While the chemistry of the nanotube exterior has been extensively studied, ${ }^{6}$ the reactivity of its interior still remains largely unexplored. We investigate the behavior of three transition metals, $\mathrm{W}, \mathrm{Re}$, and Os, encapsulated within the nanotube cavity as clusters of 20-60 atoms. Aberration-corrected HRTEM (AC-HRTEM) imaging enables detailed investigation of the atomic structure and dynamics of the metal-SWNT composites and simultaneously provides energy, in the form of the kinetic energy of the electron beam (e-beam), for activating chemical transformations in the specimen. ${ }^{7}$ The kinetic energy transferred from the e-beam to an atom due to an elastic collision $\left(E_{\text {transfer }}\right.$ calculated using the McKinsley and Feshbach approach ${ }^{8}$ ) can eject that atom from the molecule it is bound in if $E_{\text {transfer }}$ is greater than the activation energy for the dissociation of chemical bonds holding it in place. The value of $E_{\text {transfer }}$ is inversely proportional to the atomic weight of the particular element, and since the mass of an incident electron is much lower than that of an atom, it represents only a small fraction of the kinetic energy of the e-beam. In addition to the elastic collisions, the e-beam can interact inelastically with atoms and molecules causing ionization by removing electrons from their orbitals. Because such ionization is triggered by electronelectron collisions, the kinetic energy of the e-beam can in principle be transferred to the valence electrons of the specimen in its entirety. Therefore, any atomic or molecular orbitals with ionization potentials (IP) below the energy of the e-beam can be subjected to ionization. ${ }^{7}$ However, ionized atoms confined within SWNT are in direct contact with a virtually infinite supply of delocalized electrons, and thus can rapidly become reduced by withdrawing free electrons from the nanotube. Being an excellent electronic conductor, SWNT is not ionized by the e-beam, and it also prevents extensive ionization of the encapsulated molecules and atoms, as discussed below.

The energy of the e-beam can be precisely controlled by using different accelerating voltages. At a standard energy of 200-300 keV, typically used in conventional HRTEM experiments, $E_{\text {transfer }}$ to a carbon atom exceeds the energy barrier for $\mathrm{C}$-atoms escaping from the nanotube $\left(E_{\text {escape }}\right)$, resulting in rapid damage to the nanotube structure before any imaging of chemical reactions can be made. ${ }^{9}$ However, if the energy of the e-beam is decreased to $80 \mathrm{keV}$ or lower, the maximum transferable energy from an incident electron to a Catom of the nanotube $\left(\max \left(E_{\text {transfer }}\right) \leq 15.7 \mathrm{eV}\right)$ falls below the energy required to remove $\mathrm{C}$-atoms from the nanotube wall $\left(\min \left(E_{\text {escape }}\right)=17 \mathrm{eV}\right.$ for typical carbon nanotubes), ensuring that no damage is caused to the pristine SWNT structure under these conditions. It is important to note that carbon nanotubes are excellent thermal and electronic conductors. Therefore, considering the fact that the e-beam irradiates only a tiny segment of a very thin $(1-2 \mathrm{~nm})$ and extremely long nanotube (up to tens of $\mu \mathrm{m}$ ), no significant ionization of SWNT can take place. Guided by these considerations, we have carried out our measurements at 80 and $20 \mathrm{keV}$ so that pristine SWNTs remain virtually unchanged, and therefore, any transformations observed in this study can be attributed to the interactions and reactions between the nanotubes and transition metals.

Metal clusters were generated inside nanotubes by decomposing corresponding carbonyl complexes. At the beginning of the observations, all three metals appear to be physisorbed onto the internal surface of the nanotube with a clear van der Waals gap of $0.3-0.4 \mathrm{~nm}$ between the metal and the SWNT surface (Figures 1a,b and 2a). Metal clusters appear

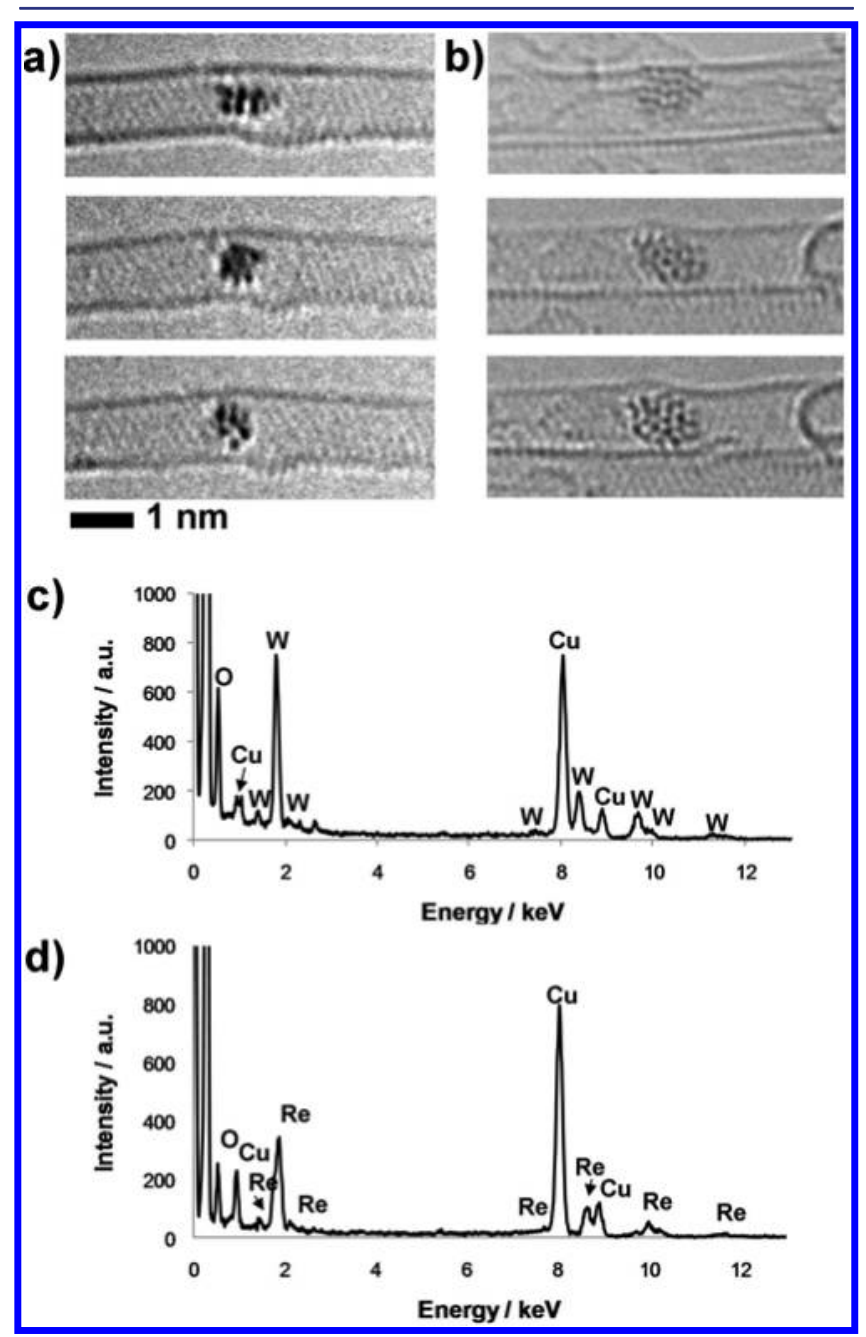

Figure 1. Time series of AC-HRTEM images showing the typical behaviors of $\mathrm{W}$ (a) and $\mathrm{Re}$ (b) clusters encapsulated in nanotubes exposed to the $80 \mathrm{keV}$ electron beam. Note that the $\mathrm{W}$ cluster exhibits only minimal interactions with the inner surface of the SWNT thus remaining centered, close to the nanotube axis, whereas the Re cluster adheres to the sidewall. The dynamics of cluster motion can be watched in Supporting Information video files. EDX spectroscopy confirms the identity of the metal clusters within the SWNT as W (c) and $\mathrm{Re}(\mathrm{d}) ; \mathrm{Cu}$-peaks are due to the TEM specimen grid.

to be moving rapidly (translation and rotation) within the nanotube cavity, thus, indicating that no covalent bonding initially exists between $\mathrm{W}, \mathrm{Re}$, or $\mathrm{Os}$ and the nanotube (Supporting Information video files). However, as the dose of the $80 \mathrm{keV}$ e-beam increases, these three metals exhibit qualitatively different interactions with the nanotube sidewalls.

Tungsten clusters remain largely unchanged throughout the entire experiment (Figure 1a). They stay positioned in the middle of the nanotube channel and tumble continuously (Supporting Information video files) indicating that the clusters 


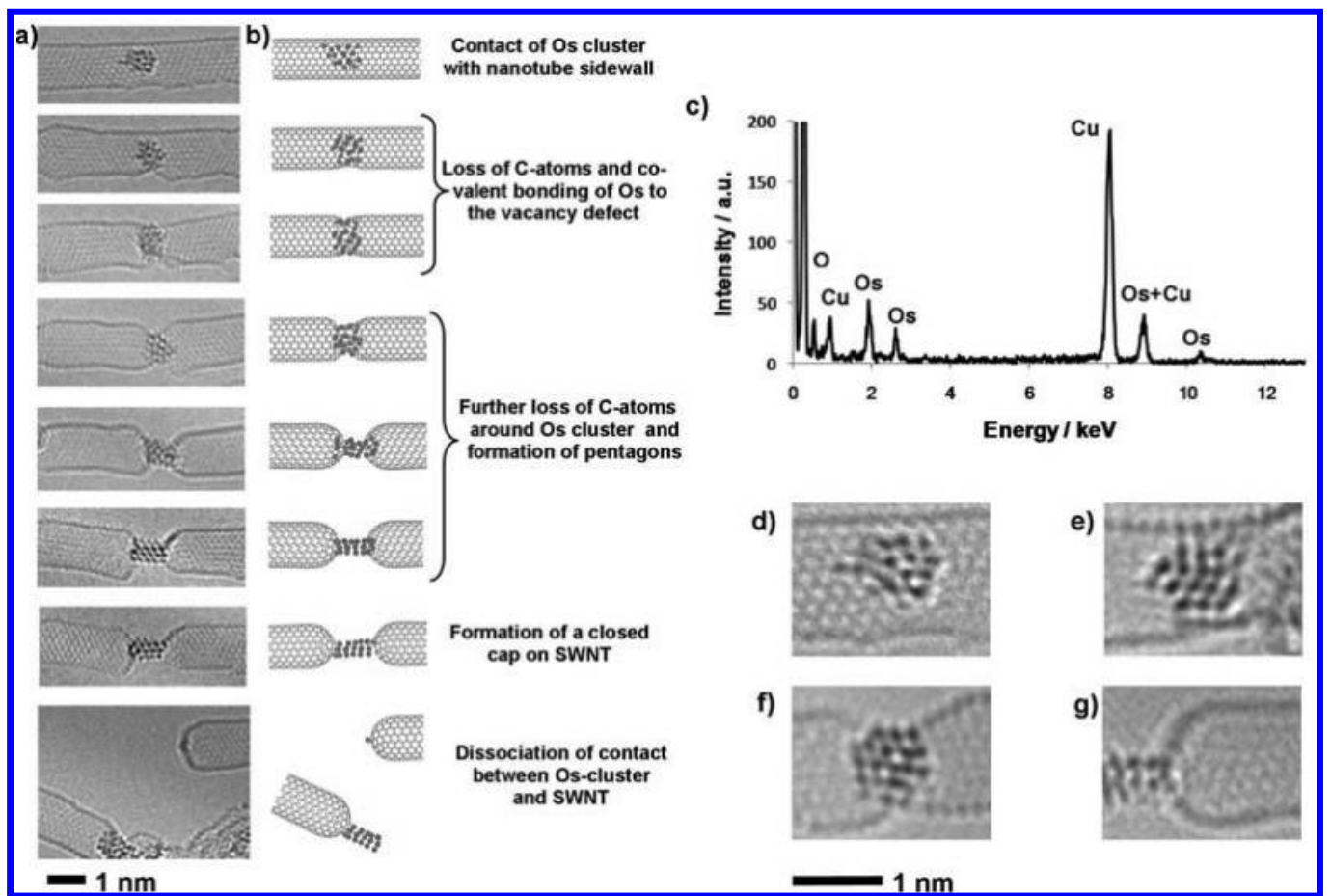

Figure 2. Stages of the reaction between an Os-cluster and SWNT imaged by AC-HRTEM at $80 \mathrm{keV}$ (a) with (b) the corresponding structural diagrams (numbers of metal atoms in diagrams are reduced for the sake of clarity). EDX spectroscopy confirms the identity of the metal clusters within the nanotubes as Os (c); Cu-peaks are due to the TEM specimen grid. High-magnification images showing details of key steps: $\pi$-bonding between an Os-cluster and the interior of the SWNT (d and e), $\sigma$-bonding between an Os-cluster and a defect on the SWNT (f), and $\pi$-bonding between an Os-cluster and the exterior of the SWNT (g).

remain physisorbed on the SWNT interior surface without forming any directional bonding with the surrounding carbon atoms. As a result, the nanotube sidewalls in the vicinity of $\mathrm{W}$ clusters remain largely unchanged for the entire duration of the experiment.

Rhenium clusters appear to be more reactive toward the nanotube (Figure 1b). As the dose of the e-beam increases, Re clusters adhere to the sidewalls of the SWNT, forming a much closer contact of $\sim 0.2 \mathrm{~nm}$, which is shorter than the van der Waals gap of $0.3-0.4 \mathrm{~nm}$ observed initially. Stronger interactions with the Re cluster distort the nanotube sidewall, forming undulations and vacancy defects. This clearly indicates that the $\mathrm{C}-\mathrm{C}$ bonds of the nanotube are substantially perturbed by this metal. After prolonged irradiation with the $80 \mathrm{keV}$ e-beam, some Re clusters appear to protrude through the sidewall (Supporting Information video files). However, the defects created by Re stay localized and do not affect the overall integrity of the SWNT structure.

Osmium clusters are most reactive toward the nanotube (Figure 2). They adhere strongly to the SWNT causing distortion of the sidewall. The degree of distortion in the vicinity of the metal clusters increases steadily, while the surface atoms of the Os-cluster appear to be inserted into vacancy defects developing around them. The entire section of the nanotube around the Os particle appears to be shrinking as those carbon atoms, which are in contact with the cluster, become removed by the e-beam. Once the metal surface is exposed, the cluster interacts strongly with the dangling carbon bonds of the nanotube defect and spans the two parts of the severed nanotube (Figure 2). The metal atoms then rearrange to form parallel chains of 6-7 atoms long, while the open edges of the nanotubes start closing down. The topology of a closed SWNT cap demands the formation of six pentagons, which is driven by the thermodynamic requirement for the minimization of the number of dangling carbon bonds in the structure. Once the cap is fully closed, all dangling bonds are eliminated and the interactions between the $\mathrm{Os}$ and the nanotube appear to weaken leading to the detachment of the metal cluster from the closed cap of the nanotube (Figure 2).

This remarkable difference in the reactivity of the three transition metals is highly consistent across different samples (in total, 12 different samples were prepared and studied) and different TEM conditions (three different TEM instruments operated at different accelerating voltages were employed; for details see Section S1 of the Supporting Information). Most significantly, the processes described above for $\mathrm{W}, \mathrm{Re}$, and Os appear to be independent of the size or shape of the metal clusters. Typically, clusters consist of 20-60 atoms and their shapes are continuously changing (as expected for very small metallic particles at room temperature; Figures 1 and 2), but regardless of their size, shape, or initial orientation inside the nanotube, their behavior is determined by the chemical nature of the element. The exact configuration of the atoms within the clusters has only subtle effects on the observed kinetics of the interactions and reactions with the SWNT, but the final outcome of each process is strictly defined by the type of metal present.

As neighboring elements in the Periodic Table, W, Re, and Os have very similar atomic sizes, ionization potentials, and electronegativities. However, our density functional theory (DFT) calculations show that these metals have very different affinities for a SWNT, as illustrated by their binding energies to the inner surface of the nanotube (Table 1). Os forms the strongest interactions, and $\mathrm{W}$ forms the weakest. In addition, the e-beam of TEM, even at $80 \mathrm{keV}$, has sufficient energy to remove the valence electrons of the metals, since the ionization 
Table 1. DFT Parameters Describing Interactions of W, Re, or Os with the Inner Surface of $1 \mathrm{~nm}$ Long $(10,10)-S W N T$ in Periodic Boundary Conditions

\begin{tabular}{cccc} 
system & $\begin{array}{c}\text { binding } \\
\text { energy } \\
(\mathrm{eV})\end{array}$ & $\begin{array}{c}\text { electronic density } \\
\text { removed by } \mathrm{M}^{+} \text {from } \\
\text { SWNT (electrons) }\end{array}$ & $\begin{array}{c}\text { electronic density } \\
\text { removed by } \mathrm{M}^{2+} \text { from } \\
\text { SWNT (electrons) }\end{array}$ \\
$\mathrm{W} @(10,10)$ & 0.030 & 0.09 & 1.02 \\
$\mathrm{Re}(10,10)$ & 0.046 & 0.11 & 1.09 \\
$\mathrm{Os@(10,10)}$ & 0.238 & 0.37 & 1.31 \\
\hline
\end{tabular}

potentials for the outer electronic shell of these metals are all below $0.1 \mathrm{keV} .^{10}$ Therefore, the e-beam can be viewed as an oxidant that can potentially increase the oxidation state of the metals. However, being confined within the nanotube, the metal clusters are in intimate contact with the SWNT sidewall, thus, allowing electronic density exchange to occur readily between the metal cations and the SWNT electronic bands (Scheme 1a). Our calculations show that ionized atoms $\mathrm{W}^{n+}$, $\mathrm{Re}^{n+}$, and $\mathrm{Os}^{n+}$ withdraw $m$ electrons from a SWNT container (Scheme 1a). Once again, the chemical nature of the element determines the amount of charge transferred from the SWNT onto the metal cation, with Os cations abstracting the highest amount of electronic density (Table 1). Both parameters, the binding energy and the charge redistribution, indicate that the interactions between the metals and nanotube increase in the order $\mathrm{W}<\mathrm{Re}<\mathrm{Os}$, which correlates directly with the relative reactivities of these elements observed in our AC-HRTEM measurements. A recent theoretical investigation predicted that light transition metals $\mathrm{Cr}-\mathrm{Mn}-\mathrm{Fe}$ belonging to the same groups of elements as $\mathrm{W}-\mathrm{Re}-\mathrm{Os}$ should exhibit a similar order of bonding efficiency to the exterior of the $\mathrm{SWNT}^{3 \mathrm{~d}}$ (no prediction for the affinity toward the nanotube interior was made).

In our experiments, the observed metal-metal distances within the clusters are close to those in the corresponding bulk crystalline metals. This indicates that most of the metal atoms remain charge-neutral, while the surface atoms may change their oxidation state transiently under the influence of the e-beam and abstract electrons from SWNT sidewall as described above. The coupling of $d$-orbitals of the outermost metal atoms with $\pi$-orbitals of the SWNT (Scheme 1b) and nanotube-to-metal electron redistribution will both have an activating effect on the nanotube sidewall. For instance, it is well-known that the main consequence of effective $\pi$-bonding of transition metal atoms to aromatic compounds is stretching and weakening the $\mathrm{C}-\mathrm{C}$ bonds of the ligand molecules making them more reactive toward other reagents. ${ }^{11}$ Examples from organometallic synthetic chemistry suggest that Os activates aromatic molecules, such as benzene, to a significantly greater degree than $\mathrm{W}$ or Re under the same conditions. ${ }^{12}$ In our case, a SWNT acts as a nanosized polyaromatic ligand, so that metal atoms coordinated to the inner surface of the nanotube enhance the reactivity of the $\mathrm{C}-\mathrm{C}$ bonds in the vicinity of the clusters. Thus, activated sections of the SWNT become more susceptible to ballistic ejection by the e-beam, as evidenced by a gradual removal of carbon atoms around Os (Figure 2 and Scheme 1b).

Our DFT calculations show that the presence of an $\mathrm{Os}_{55}$-cluster in the nanotube lowers the $\min \left(E_{\text {escape }}\right)$ to 11.9 $\mathrm{eV}$ making it well below the maximum transferable energy from the $80 \mathrm{keV}$ e-beam to a carbon atom $\left(\max \left(E_{\text {transfer }}\right)=15.7 \mathrm{eV}\right)$. This means that the inequality $\min \left(E_{\text {escape }}\right)>\max \left(E_{\text {transfer }}\right)$, which ensures the structural integrity of SWNT under the 80 $\mathrm{keV}$ e-beam, inverts to $\min \left(E_{\text {escape }}\right)<\max \left(E_{\text {transfer }}\right)$ for the sections of SWNT engaged in $\pi$-bonding with Os, which allows the removal of carbon atoms in the vicinity of the Os-cluster and triggers the sequence of transformations observed by ACHRTEM imaging (Figure 2). To verify this mechanism, the energy of the e-beam was reduced to $20 \mathrm{keV}$, at which $\max \left(E_{\text {transfer }}\right)$ from the e-beam to a C-atom is $3.73 \mathrm{eV}$, significantly below the $\min \left(E_{\text {escape }}\right)$, even when the SWNT is activated by a Os-cluster. Using the electron dose of $10^{10} \mathrm{e} / \mathrm{nm}^{2}$ in a control AC-HRTEM experiment at $20 \mathrm{keV}$ revealed that Os-clusters adhere to the nanotube, indicating metal-carbon $\pi$ -

Scheme $1^{a}$

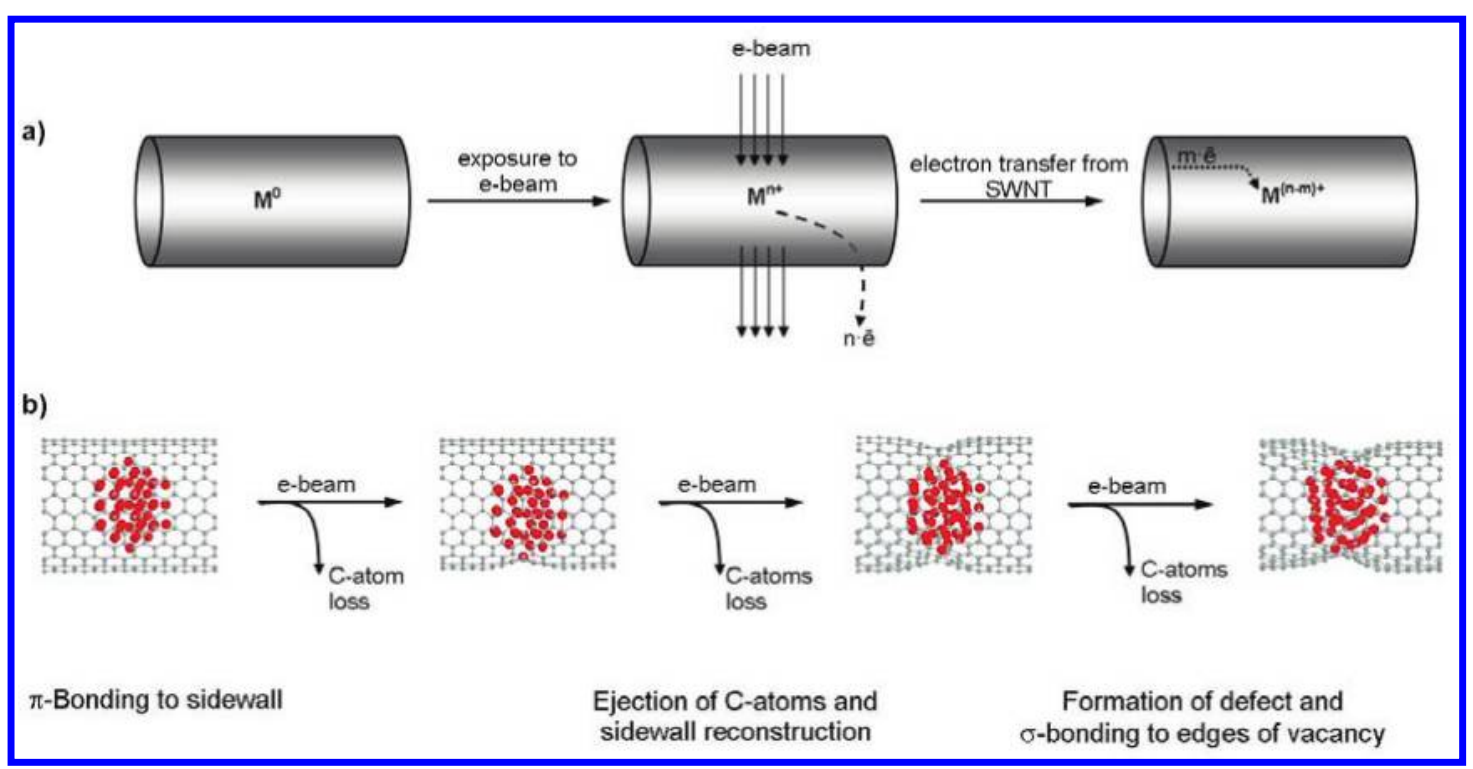

${ }^{a}$ The effective oxidation state and the number of valence $d$-electrons on the outermost atoms of a metal cluster are determined by the balance of valence electron removal by the e-beam and the electron density redistribution (a). DFT optimized structures showing key stages of a mechanism for metal-catalysed transformations in SWNT calculated for $\mathrm{Os}_{55}$-cluster inside $(12,12)$-SWNT (b). 
bonding, but no significant sidewall defects emerge in the vicinity of the clusters at $20 \mathrm{keV}$ (Supporting Information). The same dose of $80 \mathrm{keV}$ electrons causes extensive structural transformations culminating in a complete rupture of the SWNT activated by Os-cluster, emphasizing the importance of the energy of incident electrons for nanotube-metal bonding.

As the e-beam removes carbon atoms and creates a vacancy defect around the Os-cluster, the nanotube atoms at the edge of the defect form $\sigma$-bonds with the metal (Figure 3a) with typical

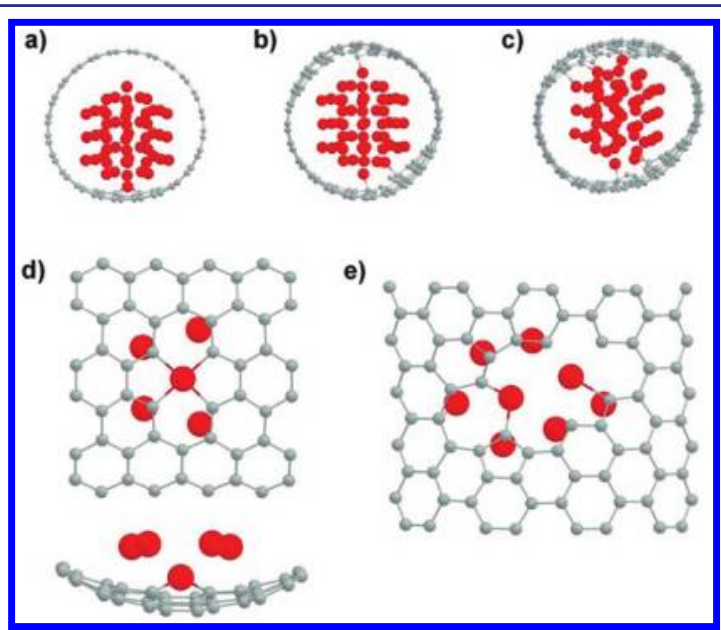

Figure 3. DFT optimized structures illustrating the stages of $\mathrm{Os}_{55}$-cluster binding to defects of SWNT $(\mathrm{a}-\mathrm{c})$. The nanotube cylindrical shape becomes progressively distorted as the size of defect increases and the number of metal-carbon $\sigma$-bonds becomes greater. Plane-view diagrams of different binding sites ( $\mathrm{d}$ and e) (only surface Os atoms which engage in bonding with the defect are shown, and the rest of atoms of Os-cluster are omitted for clarity).

metal-carbon bond lengths of $0.20-0.21 \mathrm{~nm}$. The formation of effective $\sigma$-bonds between the nanotube and the metal is responsible for bringing clusters progressively closer to the nanotube surface over time, as observed in AC-HRTEM experiments (Figures 1 and $2 \mathrm{~d}-\mathrm{g}$ ). As the defect grows, the number of carbon-metal $\sigma$-bonds increases and the nanotube shape distorts further away from the cylindrical shape of a pristine SWNT (Figure $3 \mathrm{a}-\mathrm{c}$ ). At the stage of a di-vacancy defect, one of the Os-atoms of metal cluster forms $\sigma$-bonds with four carbon atoms and becomes effectively inserted into the atomic lattice of the SWNT (Figure 3d). This step initiates a process of metal cluster protrusion through the nanotube sidewall experimentally observed for Os (Figure 2). It is interesting to note that the HRTEM images simulated from theoretical models predict that structural transformations in the SWNT become really noticeable only after six or more carbon atoms are removed from its sidewall (Supporting Information). This means that at the point when distortions in the SWNT become observable in AC-HRTEM experiments, extensive multiatom vacancies are likely to be formed on the nanotube.

A number of reports demonstrated a significantly stronger bonding of metal atoms to vacancy defects in graphene as compared to a pristine graphene structure. ${ }^{13} \mathrm{~A}$ recent experimental study has confirmed that transition metals can become trapped in defects generated by a $200 \mathrm{keV}$ e-beam in multiwalled carbon nanotubes (MWNTs) due to a stronger bonding with dangling carbon bonds than with the $\pi$-electron system of an intact MWNT. ${ }^{14}$ Clearly, the presence of $\sigma$ bonding of metal to carbon atoms around the defect edge must provide some stabilization to the SWNT, as $\mathrm{M}-\mathrm{C}$ bonds terminate the highly reactive dangling bonds. Our calculations of the density of states (DoS) for a $(12,12)$-SWNT show that implantation of metal clusters into the nanotube sidewall perturbs the electronic structure of the SWNT quite significantly, due to a strong hybridization between the $d$ orbitals of the Os-cluster and the $\pi$-electronic system of the host-nanotube (Figure 4).

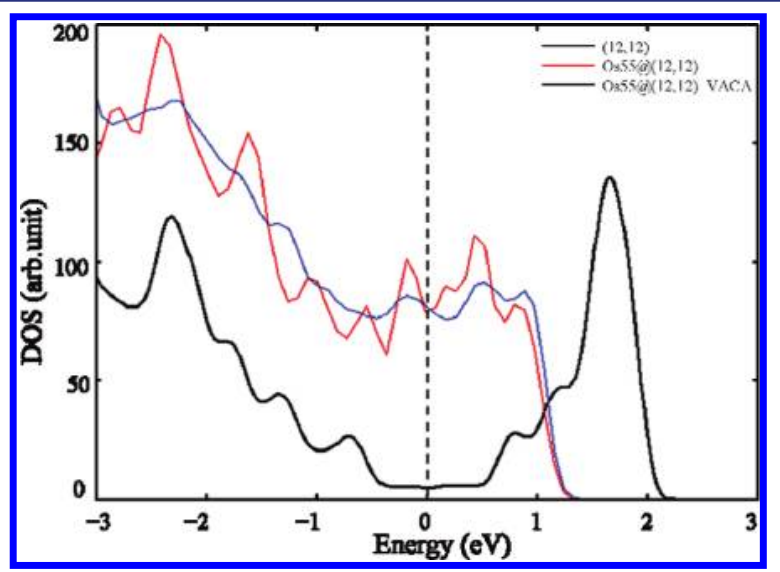

Figure 4. Density of states plots for (black) a pristine $(12,12)-S W N T$, (red) $\mathrm{Os}_{55}$ inside $(12,12)$-SWNT, and (blue) $\mathrm{Os}_{55}$ inside $(12,12)$ SWNT with defects ( 12 carbon atoms missing). The zero corresponds to the Fermi energy.

Since metal-carbon bonds are more labile than $\mathrm{C}-\mathrm{C}$ bonds, the thermodynamically most stable bonding configuration ultimately corresponds to a closed cap where each carbon atom forms three bonds with other C-atoms. We have previously demonstrated that the formation of closed graphitic structures is facilitated by the e-beam through the loss of edge carbon atoms and the formation of pentagons providing the curvature required for a closed structure. ${ }^{5 \mathrm{~T}}$ DFT calculations clearly show that even at the stage of six-vacancy (Figure 3e) the neighboring carbon atoms already start recombining their dangling bonds to form pentagons, which is the first step of the SWNT cap formation process. Our experimental AC-HRTEM observations show that from this point the nanotube defect reconstructs extensively until a fully sealed cap, with no dangling bonds and six pentagons (required by the topology of a closed cap), is formed (Figure 2g). Once the metal cluster is detached from the nanotube, no further transformations take place in the SWNT structure.

\section{CONCLUSIONS}

Understanding the physicochemical interactions and bonding between carbon nanostructures and transition metals is of paramount importance for further technological applications of these materials. In this study, we have demonstrated and compared the reactivity of different transition metals with SWNTs at different electron beam energies. The interactions and chemical reactions between $\mathrm{W}, \mathrm{Re}$, and Os, separately, and the SWNT were followed in real time and direct space at the atomic level by $80 \mathrm{keV}$ AC-HRTEM. By confining the metal clusters inside a SWNT, it is possible to image the metals "in action" revealing their very different reactivities, in the order $\mathrm{W}<\mathrm{Re}<\mathrm{Os}$, toward the nanotube at this electron beam energy. The observed order is not dependent on size, shape, or orientation of metal clusters. The greater reactivity of Os is 
largely related to the more efficient binding of this metal to SWNT as compared to other metals. The recently demonstrated capability of high resolution TEM for imaging electron density distribution in structures containing carbon and nitrogen ${ }^{15}$ can in the future be extended to organometallic systems to reveal the complex nature of nanotube-metal bonding at the subatomic level. If the rationale of organometallic chemistry previously developed for molecular compounds is applied for the interpretation of the bonding and reactivity between transition metals and carbon nanotubes, with the latter acting as nanosized polyaromatic ligands, late transition metals, whose chemistry is similar to that of Os, will exhibit a strong reactivity toward SWNT. Further exploration of the mechanisms of interactions between metals and nanotubes has the potential to open new ways of synthesis and manipulation of carbon nanostructures, and harnessing their functional properties.

\section{EXPERIMENTAL SECTION}

Materials. Arc-discharge SWNTs were annealed in air at $520{ }^{\circ} \mathrm{C}$ for $30 \mathrm{~min}$ to open their termini prior to addition of metal carbonyls. A mixture of SWNT and a metal carbonyl was heated in vacuum at $10{ }^{\circ} \mathrm{C}$ above the vaporization temperature of the molecules. The metal carbonyl complexes spontaneously decompose into metal clusters inside the nanotubes either thermally (i.e., by heating above their decomposition temperature) or under the e-beam of TEM. In both cases, small clusters of metals are formed within SWNTs.

Electron Microscopy. Carbon nanotubes filled with W, Re, or Os were dispersed in methanol using an ultrasonic bath, drop-cast onto lacey-carbon coated copper TEM grids (Agar) and heated at $150{ }^{\circ} \mathrm{C}$ for $5 \mathrm{~min}$ before insertion into the TEM column. Conventional HRTEM imaging and local energy dispersive X-ray spectroscopy (EDX) were carried on a JEOL $2100 \mathrm{~F}$ operated at $100 \mathrm{kV}$. Aberration corrected imaging and e-beam irradiation experiments were carried out on a $\mathrm{C}_{\mathrm{S}}$-corrected FEI Titan 80-300 TEM operated at $80 \mathrm{kV}$ with information limit enhancement by reduced extraction voltage ${ }^{16}$ or a Zeiss Libra 200MC operated at $80 \mathrm{kV}$ where an information limit shift is provided by a monochromator $(0.15 \mathrm{eV})$, and contrast is enhanced by zero-loss energy filtering $(5 \mathrm{eV})$. For $20 \mathrm{keV}$ AC-HRTEM experiments, special modifications were applied regarding the corrector and base setup. ${ }^{17}$ All measurements were performed at room temperature, and the electron flux applied to the samples ranged from $2 \times 10^{6}$ to $7 \times 10^{6} \mathrm{e}^{-} /\left(\mathrm{nm}^{2} / \mathrm{s}\right)$.

Computational Details. First-principles density functional theory calculations of the optimized structures and densities of states of a $(10,10)$-SWNT containing W, Re, and Os transition metal atoms and a $(12,12)$-SWNT containing an $\mathrm{Os}_{55}$-cluster have been performed using the CASTEP code. ${ }^{18}$ The exchange and correlation interactions are described using generalized gradient approximation (GGA) parametrized by Perdew, Burke, and Ernzerhof (PBE), ${ }^{19}$ and ultrasoft pseudopotentials are generated "on-the-fly". A plane wave basis set with the energy cutoff of $320 \mathrm{eV}$ is used. In the optimized structures, the forces on atoms are smaller than $0.05 \mathrm{eV} / \mathrm{A}$. For the $\mathrm{Os}_{55} @(12,12)$ SWNT system, a supercell with 25,25 , and $22.5 \AA$ dimensions along $x$, $y$, and $z$ directions, respectively, is used, and for the metal atom inside $(10,10)$-SWNT, a $(30 \times 30 \times 9.98) \AA^{3}$ supercell is used. This allows SWNTs to have their periodicity preserved along the $z$-axis, while eliminating the interactions between clusters and their periodic images. A single $k$ point $(\Gamma)$ was used in all calculations.

\section{ASSOCIATED CONTENT}

\section{S Supporting Information}

Observation of metal clusters in SWNT using $20 \mathrm{keV}$ e-beam; observation of metal clusters in SWNT; videos illustrating the behavior of each metal (AVI-files). This material is available free of charge via the Internet at http://pubs.acs.org.

\section{AUTHOR INFORMATION}

\section{Corresponding Author}

Elena.Bichoutskaia@nottingham.ac.uk; Ute.Kaiser@uni-ulm.de; Andrei.Khlobystov@nottingham.ac.uk

\section{ACKNOWLEDGMENTS}

This work was supported by the DFG (German Research Foundation) and the Ministry of Science, Research and the Arts (MWK) of Baden-Württemberg in the frame of the SALVE (Sub Angstrom Low-Voltage Electron microscopy project) and by the DFG within the research project SFB 569 (T.Z., U.K., and J.B.); an EPSRC Career Acceleration Fellowship and "NanoTP" COST action (E.B.) and High Performance Computing (HPC) facility at the University of Nottingham (E.B. and N.K.); the EPSRC, ESF and the Royal Society (T.W.C. and A.N.K.); and the Nottingham Nanoscience and Nanotechnology Centre (access to the Jeol 2100F instrument).

\section{REFERENCES}

(1) (a) Tian, W. Q.; Liu, L. V.; Yang, Y. A. Phys. Chem. Chem. Phys. 2006, 8, 3528-3539. (b) Kong, K.; Choi, Y.; Ryu, B.; Lee, J.; Chang, H. Mater. Sci. Eng. C 2006, 26, 1207-1210. (c) Pan, X.; Bao, X. Chem. Commun. 2008, 6271-6281. (d) Castillejos, E.; Debouttiere, P. J.; Roiban, L.; Solhy, A.; Martinez, V.; Kihn, Y.; Ersen, O.; Philippot, K.; Chaudret, B.; Serp, P. Angew. Chem., Int. Ed. 2009, 48, 2529-2533.

(2) (a) Yildrim, T.; Ciraci, S. Phys. Rev. Lett. 2005, 94, 175501.

(3) (a) Dong, X.; Lau, C. M.; Lohani, A.; Mhaisalkar, S. G.; Kasim, J.; Shen, Z. X.; Ho, X.; Rogers, J. A.; Li, L.-I. Adv. Mater. 2008, 20, 23892392. (b) Nemec, N.; Tomanek, D.; Cuniberti, G. Phys. Rev. Lett. 2006, 96, 076802. (c) Palacios, J. J.; Perez-Jimenez, A. J.; Louis, E.; SanFabian, E.; Verges, J. A. Phvs. Rev. Lett. 2003, 90, 106801. (d) Yang, C. K.; Zhao, J.; Lu, J. P. Nano Lett. 2004, 4, 561-563. (e) RodriguesManzo, J. A.; Banhart, F.; Terrones, M.; Terrones, H.; Grobert, N.; Ajayan, P. M.; Sumpter, B. G.; Meunier, V.; Wang, M.; Bando, Y.; Goldberg, D. Proc. Natl. Acad. Sci. U.S.A. 2009, 106, 4591-4595.

(4) (a) Yang, S. H.; Shin, W. H.; Lee, J. W.; Kim, S. Y.; Woo, S. I.; Kang, J. K. I. Phvs. Chem. B 2006, 110, 13941-13946. (b) Zhuang, H. L.; Zheng, G. P.; Soh, A. K. Comput. Mater. Sci. 2008, 43, 823-828. (c) Valencia, H.; Gil, A.; Frapper, G. I. Phys. Chem. C 2010, 114, 14141-14153. (d) Inntam, C.; Limtrakul, J. J. Phys. Chem. C 2010, 114, 21327-21337. (e) Chen, Y. K.; Liu, L. V.; Tian, W. Q.; Wang, Y. A. I. Phvs. Chem. C 2011, 115, 9306-9311.

(5) (a) Nakamura, E.; Koshino, M.; Saito, T.; Niimi, Y.; Suenaga, K.; Matsuo, Y. I. Am. Chem. Soc. 2011, 133, 14151-14153. (b) Koshino, M.; Tanaka, T.; Solin, N.; Suenaga, K.; Isobe, H.; Nakamura, E. Science 2007, 316, 853. (c) Koshino, M.; Niimi, Y.; Nakamura, E.; Kataura, H.; Okazaki, T.; Suenaga, K; Iijima, S. Nat. Chem. 2010, 2, 117-124. (d) Chuvilin, A.; Bichoutskaia, E.; Gimenez-Lopez, M. C.; Chamberlain, T. W.; Rance, G. A.; Kuganathan, N.; Biskupek, J.; Kaiser, U.; Khlobystov, A. N. Nat. Mater. 2011, 10, 687-692. (e) Chamberlain, T. W.; Meyer, J. C.; Biskupek, J.; Leschner, J.; Santana, A.; Besley, N. A.; Bichoutskaia, E.; Kaiser, U; Khlobystov, A. N. Nat. Chem. 2011, 3, 732-737. (f) Chuvilin, A.; Kaiser, U.; Bichoutskaia, E.; Besley, N. A.; Khlobystov, A. N. Nat. Chem. 2010, 2, 450-453.

(6) Tasis, D.; Tagmatarchis, N.; Bianco, A.; Prato, M. Chem. Rev. 2006, 106, 1105-1136.

(7) Williams, D. B.; Carter, C. B. Transmission Electron Microscopy: A Textbook for Materials Science, 2nd ed.; Springer: New York, 2009.

(8) McKinsley, W.; Feshbach, H. Phvs. Rev. 1948, 74, 1759.

(9) (a) Smith, B. W.; Luzzi, D. E. I. Appl. Phvs. 2001, 90, 3509-3515. (b) Zobelli, A.; Gloter, A.; Ewels, C. P.; Seifert, G.; Colliex, C. Phys. Rev. B 2007, 75, 245402.

(10) CRC Handbook of Chemistry and Physics, 86th ed.; Lide, D. R., Ed.; CRC Press: Boca Raton, FL, 2005.

(11) Muetterties, E. L.; Bleeke, J. R.; Wucherer, J.; Albright, T. A. Chem. Rev. 1982, 82, 499-525. 
(12) Ding, F.; Harman, W. D. I. Am. Chem. Soc. 2004, 126, 1375213756.

(13) (a) Krasheninnikov, A. V.; Lehtinen, P. O.; Foster, A. S.; Pyykko, P.; Nieminen, R. M. Phys. Rev. Lett. 2009, 102, 126807. (b) Gan, Y.; Sun, L.; Banhart, F. Small 2008, 4, 587-591. (c) Chan, K.

T.; Neaton, J. B.; Cohen, M. L. Phvs. Rev. B 2008, 77, 235430.

(d) Anton, R.; Schneidereit, I. Phvs. Rev. B 1998, 58, 13874-13881.

(14) Rodriguez-Manzo, J. A.; Cretu, O.; Banhart, F. ACS Nano 2010, 4, 3422-3428.

(15) Meyer, J. C.; Kurasch, S.; Park, H. J.; Skakalova, V.; Kunzel, D.; Gross, A.; Chuvilin, A.; Algara-Siller, G.; Roth, S.; Iwasaki, T.; Starke, U.; Smet, J. H.; Kaiser, U. Nat. Mater. 2011, 10, 209-215.

(16) Meyer, J. C.; Chuvilin, A.; Algara-Siller, G.; Biskupek, J.; Kaiser, U. Nano Lett. 2009, 9, 2683-2689.

(17) Kaiser, U.; Biskupek, J.; Meyer, J. C.; Leschner, J.; Lechner, L.; Rose, H.; Stöger-Pollach, M.; Khlobystov, A. N.; Hartel, P.; Müller, H.; Haider, M.; Eyhusen, S.; Benner, G. Ultramicroscopy 2011, 111, 12391246.

(18) Payne, M. C.; Teter, M. P.; Allan, D. C.; Arias, T. A.; Joannopoulos, J. D. Rev. Mod. Phvs. 1992, 64, 1045-1097.

(19) Perdew, J. P.; Burke, K.; Ernzerhof, M. Phvs. Rev. Lett. 1996, 77, $3865-3868$. 